Література

[1] Pelykh S.N. Cladding rupture life control methods for a power-cycling WWER-1000 nuclear unit/S.N. Pelykh, M.V. Maksimov // Nuclear Engineering and Design. — 2011. — Vol. 241, № 8. — P. 2956-2963;

[2] Maksimov, M. V. A model of a power unit with WWER-1000 as an object of power control [Text] / M. V. Maksimov, K. V. Beglov, T. A. Tsiselskaya // works of the Odessa Polytechnic University. — Odessa, 2012. — Rel. 1(38). — P. 104-106;

[3] Медведєв Р.Б., Сангінова О.В. Оптимальне керування процесом зміни концентрації борної кислоти в теплоносії першого контуру АEC 3 ВВEР-1000 // Наукові вісті Національного технічного університету України “Київський політехнічний інститут”. - 2002. - № 2 (22). - С. 22;

[4] Харабет О.М. Вивчення класичної теорії автоматичного управління за допомогою персонального комп'ютера /О.М.Харабет. - О.:Бахва,2014. -188c.

References

[1] Pelykh S.N. Cladding rupture life control methods for a power-cycling WWER-1000 nuclear unit/S.N. Pelykh, M.V. Maksimov // Nuclear Engineering and Design. - 2011. - Vol. 241, № 8. — P. 2956-2963;

[2] Maksimov, M. V. A model of a power unit with WWER-1000 as an object of power control [Text] / M. V. Maksimov, K. V. Beglov, T. A. Tsiselskaya // works of the Odessa Polytechnic University. — Odessa, 2012. - Rel. 1(38). - P. 104-106.;

[3] Medvedev R.B., Sanginova O. Optimal process control of change of concentration of boric acid in the coolant-moderator of the first to the contour of AES from WWER- $1000 / /$ the Scientific news of the National technical university of Ukraine the "Kyiv polytechnic institute". - 2002. - № 2 (22). - C. 22;

[4] Kharabet O.M. A study of classic theory of automatic control is by means of the personal computer /of O.M. Kharabet - O. of: Bakhva, 2014. -188.

\title{
СИНТЕЗ И ИССЛЕДОВАНИЕ ЦИФРОВЫХ СИСТЕМ СУПЕРВИЗОРНОГО УПРАВЛЕНИЯ КОЛОННОЙ РЕКТИФИКАЦИИ НЕФТИ
}

Design and research of digital supervisory control systems of a crude oil distillation column

Стопакевич А.А. ${ }^{1}$, Стопакевич А. A. ${ }^{2}$ (Stopakevych Andrii, Stopakevych Oleksii)

${ }^{1}$ Одесская национальная академия связи им. Попова, Одесса,

${ }^{2}$ Одесский национальный политехнический университет, Одесса

E-mail: stopakevich@gmail.com

Copyright (C) 2014 by author and the journal "Automation technological and business - processes".

This work is licensed under the Creative Commons Attribution International License (CC BY).

http://creativecommons.org/licenses/by/4.0/

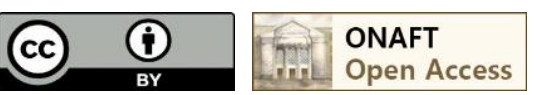

DOI: $10.15673 /$

Аннотация

В статье разрабатываются и исследуются новые системы управления нефтяной ректификационной колонной К-2 на основе нового применения супервизорного подхода. Показано, что такой подход снова распространяется в мировой практике автоматизации технологических процессов, особенно в нефтегазовой отрасли. В статье рассмотрены преимущества связки САУ на базе классических регуляторов ПИД- 
семейства и САУ на базе линейно-квадратических и МРС регуляторов. Исследовались четыре типа супервизорных систем. Анализ переходных процессов, полученных при моделировании замкнутых супервизорных систем управления при возмущении по нагрузке показывает, что все рассмотренные системы супервизорного управления повышают качество управления по сравнению с обычной децентрализованной системой на базе регуляторов ПИД-семейства и обеспечивает сопоставимое качество управления по сравнению со значительно более сложными многомерными модельно-прогнозирующими регуляторами.

\section{Abstract}

The new control systems for crude oil distillation column are developed and investigated on the base of the new usage of supervisory approach. It is shown that such approach again is spreading in world practice of technological processes automation, especially in the petroleum industry. The advantages of linking the control system on the PID-family controller base and the control system on the linear-quadratic and model predictive controller base are researched. Four types of supervisory systems were researched. Analysis of transitional processes in closed control systems in case of load disturbances shows that all researched supervisory control systems increase control quality in comparison with decentralized control systems on PID-family controller base and provide equal quality in comparison with much more complex multivariable model predictive controllers.

\section{Ключевые слова}

Ректификационная колонна, нефть, система автоматического управления, супервизорная, линейноквадратический, модельно-прогнозирующий, ПИД, регулятор

\section{Введение}

Современные требования к повышению качества продукции нефтепереработки требуют повышения качества управления нефтяными ректификационными колоннами. Особенностью таких колонн является нелинейность статических характеристик, значительная инерционность процессов, дрейф параметров во времени, значительное количество взаимосвязанных параметров. Нарушения материального баланса при отборе фракции или теплового режима в колонне приводит к изменению состава фракций и, соответственно, к изменению диапазона их температур кипения. А такие изменения ухудшают качестве готовой продукции. И, кроме того, для управления колонной целесообразно использовать параметры, связанные напрямую с материальным балансом, которые невозможно качественно регулировать независимо. В то же время, синтез систем автоматического управления (САУ) сложными многомерными и многосвязными объектами с запаздыванием является научной проблемой, для которой пока не найдено единого эффективного решения. В статье на основе разработке и анализа ряда систем управления предложена лучшая как для ректификационных колонн, так и, возможно, для других сложных многомерных и многосвязных объектов с запаздыванием.

Цель статьи

Назработка и анализ современных систем супервизорного управления ректификационными колоннами путем комбинации многомерных и классических регуляторов ПИД-семейства.

Анализ последних исследований и публикаций

В современной мировой практике создания систем управления сложными объектами снова распространяется двухуровневый подход к их структуре [1]. На первом уровне реализуется система управления на базе децентрализованных регуляторов ПИД-семейства (Distributed Control System, DCS), а на втором используются многомерные регуляторы. Ряд ведущих фирм в составе коммерческого программного обеспечения внедряет многомерные регуляторы, которые передают в качестве управляющих воздействий задания на децентрализованные регуляторы ПИД-семейства, связанные с объектом управления [2]. Исторически такой подход был обусловлен техническим разделением регуляторов - локальные автоматические регуляторы были отдельными устройствами, которые работали достаточно надежно, быстро и были проще в эксплуатации. Реализация многомерных регуляторов проводилась в компьютерной технике. Но было затруднение в ее малой вычислительной способности. Так, во внедренной в 1995 году системе управления нефтяной ректификационной колонной шаг дискретности равнялся 10 минутам [3], в то же время современная микропроцессорная техника и оптимизированные под её возможности программные алгоритмы позволяют сократить минимальный шаг дискретности до миллисекунд [4]. Современный подход к проблеме показывает, что комбинация ПИД-регуляторов и многомерных регуляторов себя хорошо рекомендует, поскольку позволяет в ряде случае упростить процедуру синтеза САУ технологическим процессом, повышает её надежность, компенсирует недостатки двух типов 


\section{МЕТОДИ ТА АЛГОРИТМИ ЕФЕКТИВНОГО УПРАВЛІННЯ ОБ‘ЭКТАМИ}

регуляторов [5]. Таким образом, данный подход остается актуальным, особенно для компьютерно интегрированного управления сложными технологическими объектами, такими как многокомпонентная нефтяная ректификационная колонна К-2 (РК) [6].

Моделирование РК. Рассмотрим РК, которая предназначена для разделения нефти со следующими свойствами: молекулярная масса - 300,00; плотность - 48,75 API (724,5 кг/м $\left.{ }^{3}\right)$; состав компонентов в газовой фазе изобутан 19\%, н-бутан $11 \%$, изопентан $37 \%$, н-пентан $46 \%$.

РК моделируется как ОУ с четырмя управляющими воздействиями ( $u_{1}-$ расход флегмы, $u_{2}-$ расход керосина в стриппинг, $u_{3}-$ расход дизеля в стриппинг, $u_{4}$ - расход газойля в стриппинг) с номиналами $50 \%$ х.и.м. и четырмя управляемыми переменными $\left(y_{1}-\right.$ уровень в конденсаторе с номиналом 50\%, $y_{2}-$ расход керосина после стриппинга с номиналом $79.3 \mathrm{~m}^{3} / \mathrm{\varphi}, y_{3}$ - расход дизеля после стриппинга с номиналом $126.5 \mathrm{~m}^{3} / \mathrm{\varphi}, y_{4}-$ расход газойля после стриппинга с номиналом $\left.14 \mathrm{~m}^{3} / \mathrm{\varphi}\right)$.

Разгонные характеристики РК, представленные на рис.1, могут быть адекватно представлены моделью высокого порядка в форме пространства состояний с шагом дискретности $\square \mathrm{t}=60 \mathrm{c}$.

$$
x_{i+1}=A_{P K} \cdot x_{i}+B_{P K} \cdot u_{i}, \quad y=C_{P K} \cdot x, \quad x=\left[x_{1}, \ldots, x_{77}\right] .
$$
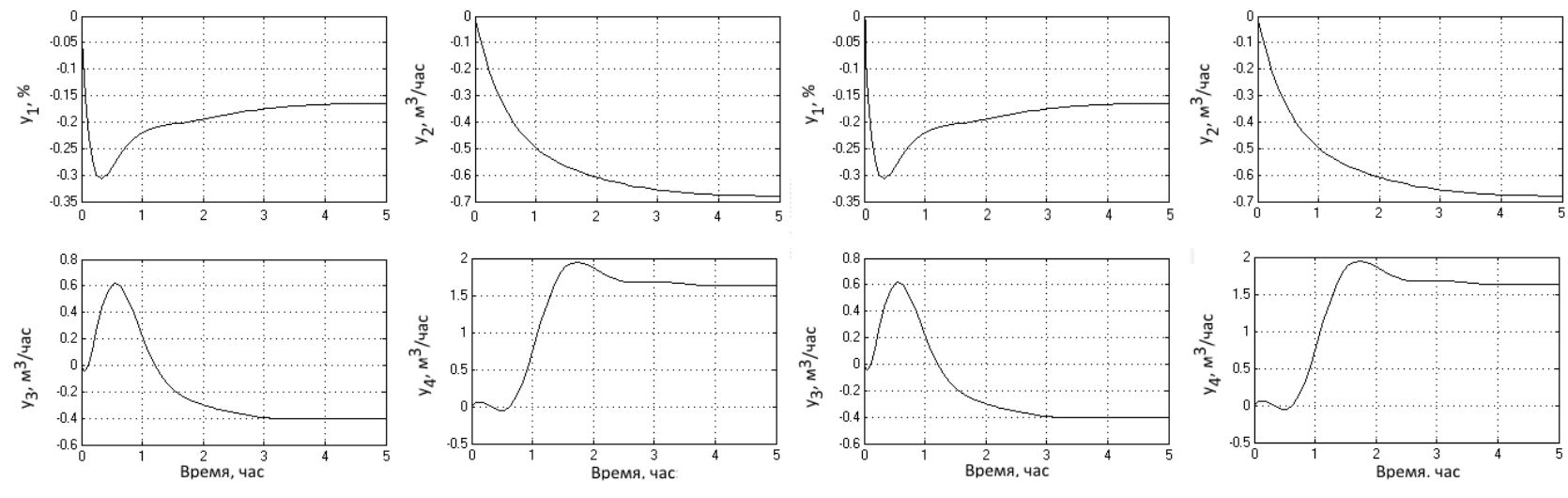

а) изменение управляющего воздействия $\mathrm{u}_{1}$ на $1 \%$

6) изменение управляющего воздействия $\mathrm{u}_{2}$ на $1 \%$
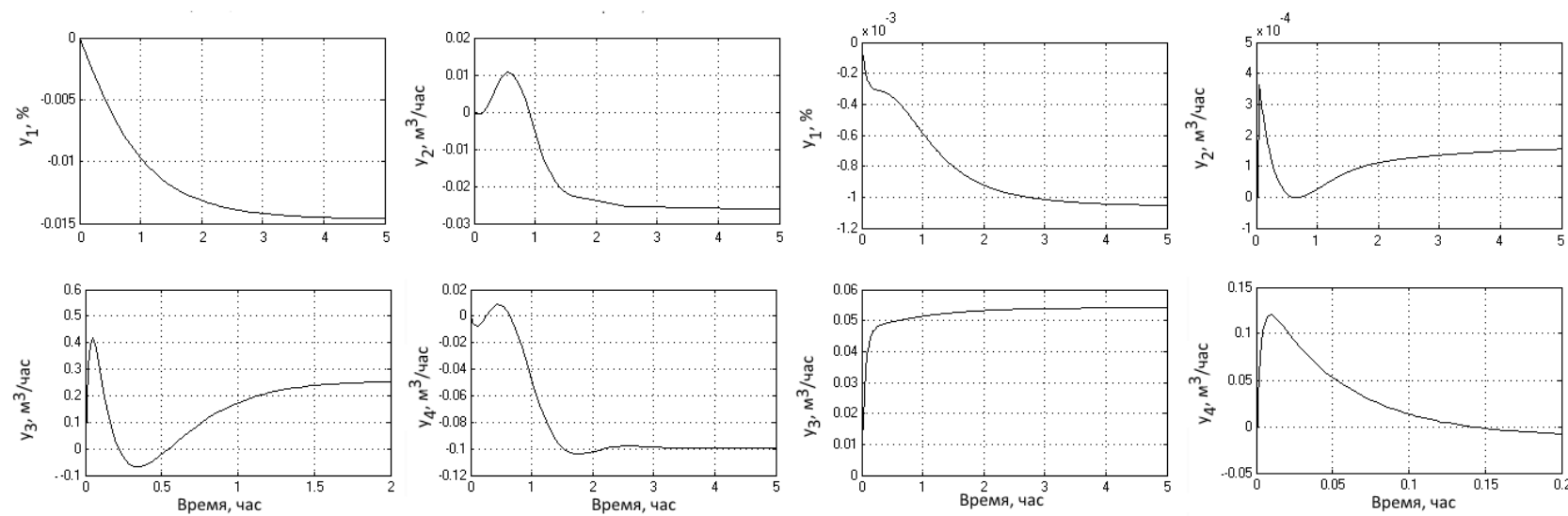

в) изменение управляющего воздействия и 3 на $1 \%$

г) изменение управляющего воздействия и 4 на $1 \%$

Рис. 1 - Разгонные характеристики РК К-2

Разгонные характеристики демонстрируют следующие особенности динамики ОУ: инерционность переходных процессов, многосвязность изменения переменных, сложную динамику каналов управления, ряд которых имеет не подходящие соотношения времени запаздывания и постоянной времени для распространенных инженерных методик настройки ПИД-регуляторов.

Постановка задачи исследования. Задачей статьи является разработка и исследование связки САУ на базе классических (ПИД-семейство) и линейно-квадратичных (LQR) регуляторов. Данная связка может быть рассмотрена как альтернатива связки САУ на базе классических и MPC (Model Predictive Control) регуляторов, внедряемой рядом ведущих фирм [7]. Разработаны и исследованы пять систем управления РК. Три системы имеют 
оригинальную структуру супервизорного управления, а именно: с передачей задания от ведущей САУ на базе LQR регулятора к ведомой на базе САУ с использованием децентрализованных ПИ-регуляторов $S_{l}$, с параллельным включением САУ на базе LQR регулятора и САУ с использованием децентрализованных ПИрегуляторов $S_{2}$, с параллельным включением ведущей САУ с использованием децентрализованных И-регуляторов и ведомой CAУ на базе LQR регулятора $S_{3}$. Для сравнения их качества разработаны супервизорная САУ с ведущей САУ на базе МРС регулятора и ведомой САУ с использованием децентрализованных ПИ-регуляторов $S_{4}$ и классическая САУ с использованием децентрализованных ПИ регуляторов $S_{0}$.

Синтез САУ с децентрализованными ПИ-регуляторами $S_{0}$. В качестве регуляторов в САУ $S_{0}$, которая может функционировать как самостоятельно, так и входит как ведомая в супервизорные системы $S_{1}$ и $S_{2}$, были выбраны ПИ регуляторы, поскольку для рассматриваемого ОУ в силу значительной инерционности качество переходных процессов в САУ на базе ПИ и ПИД регуляторов сопоставимо. Параметры цифровых ПИ регуляторов САУ были получены с помощью частотного метода, реализованного в Matlab.

Структурная схема САУ с децентрализованным цифровыми регуляторами представлена на рис.2.

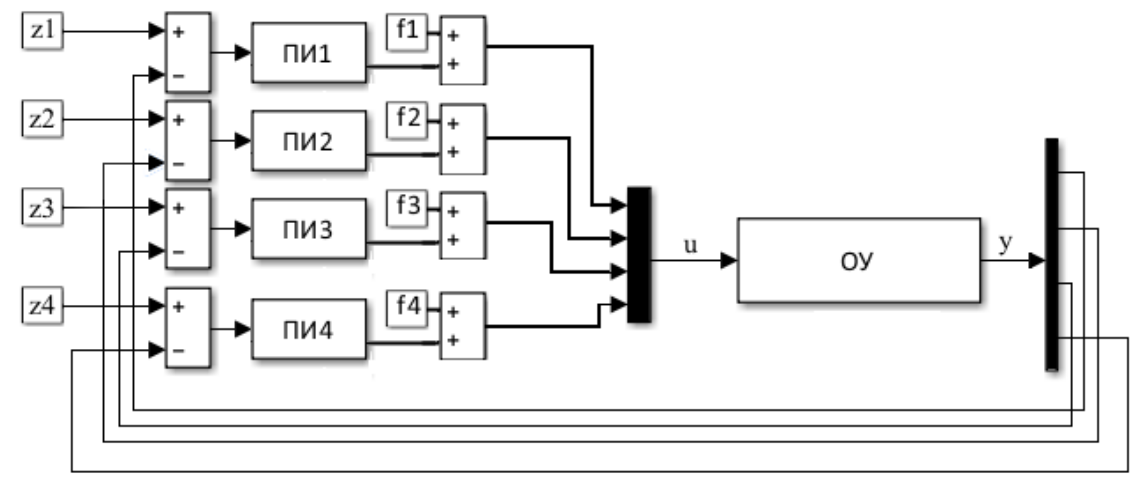

Рис. 2 - Структурная схема САУ с децентрализованным цифровыми регуляторами $S_{0}$

Модель обобщенного децентрализованного цифрового регулятора, состоящей из 4 ПИ регуляторов, имеет следующий вид

где

$$
\begin{gathered}
w_{i+1}=A_{P I} \cdot w_{i}+B_{P I}\left(z-y_{i}\right), \\
u_{i}=C_{P I} \cdot w_{i}+D_{P I} \cdot\left(z-y_{i}\right) \\
A_{P I}=\left[\begin{array}{llll}
1 & 0 & 0 & 0 \\
0 & 1 & 0 & 0 \\
0 & 0 & 1 & 0 \\
0 & 0 & 0 & 1
\end{array}\right], B_{P I}=\left[\begin{array}{cccc}
-0.5683 & 0 & 0 & 0 \\
0 & 0.0902 & 0 & 0 \\
0 & 0 & 0.0657 & 0 \\
0 & 0 & 0 & 0.1064
\end{array}\right], \\
C_{P I}=\left[\begin{array}{llll}
1 & 0 & 0 & 0 \\
0 & 1 & 0 & 0 \\
0 & 0 & 1 & 0 \\
0 & 0 & 0 & 1
\end{array}\right], D_{P I}=\left[\begin{array}{cccc}
-1.24366 & 0 & 0 & 0 \\
0 & 2.95196 & 0 & 0 \\
0 & 0 & -0.0328561 & 0 \\
0 & 0 & 0 & 0.0212817
\end{array}\right] .
\end{gathered}
$$

Супервизорная система $S_{1}$. Структурная схема системы супервизорного управления $S_{I}$ представлена на рис.3. 


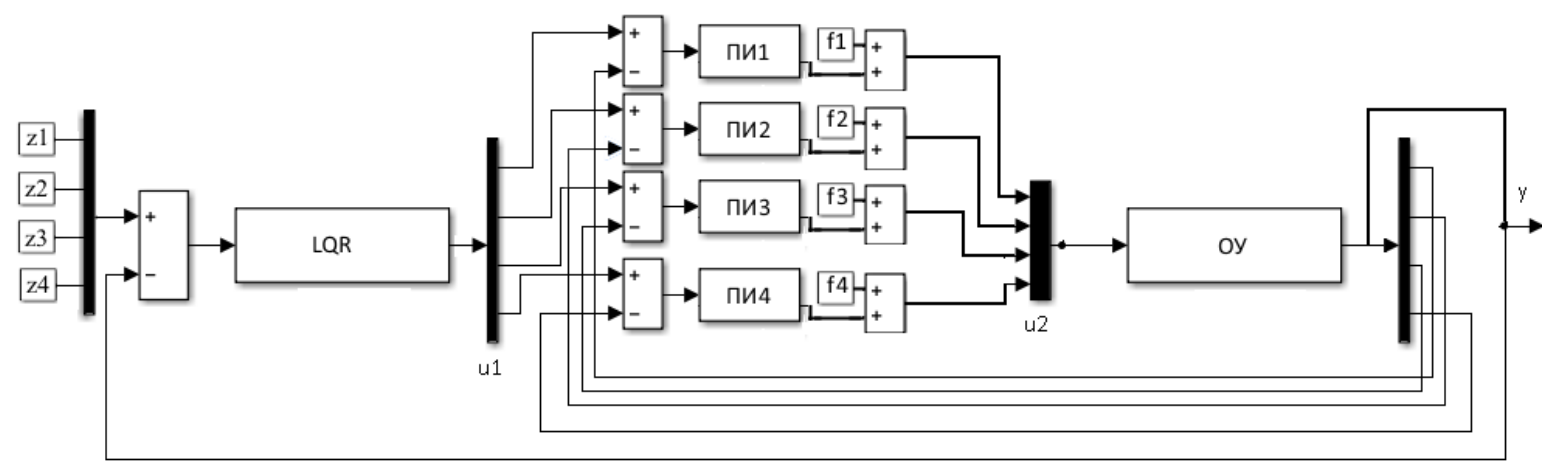

Рис. 3 - Структурная схема системы супервизорного управления $S_{l}$

Для CAУ на базе LQR-регулятора объектом управления является CAУ $S_{0}$, матрицы которой имеют вид

$$
A z_{1}=\left[\begin{array}{cc}
A_{P K}-B_{P K} \cdot D_{P I} \cdot C_{P K} & B_{P K} \cdot C_{P I} \\
-B_{P I} \cdot C_{P K} & A_{P I}
\end{array}\right], \quad B z_{1}=\left[\begin{array}{c}
B_{P K} \cdot D_{P I} \\
B_{P I}
\end{array}\right], \quad C z_{1}=\left[\begin{array}{ll}
C_{P K} & 0
\end{array}\right] .
$$

Матрицы обобщенного регулятора CAY на базе LQR-регулятора, состоящего из LQR-регулятора и наблюдателя состояния, имеют вид

$$
A r_{1}=A z_{1}-B z_{1} \cdot K-L \cdot C z_{1}, \quad B r_{1}=L, \quad C r_{1}=K, \quad D r_{1}=0
$$

где матрицы $K$ и $L$ рассчитываются с помощью стандартных Matlab - программ

$$
K=\operatorname{dlqr}\left(A z_{1}, B z_{1}, Q_{k}, R_{k}\right), \quad L=d q \operatorname{lr}\left(A z_{1}, C z_{1}{ }^{\prime}, Q_{L}, R_{L}\right)^{\prime} .
$$

Для достижения оптимального переходного процесса с минимальной статической ошибкой параметры LQRрегулятора выбраны в виде $Q_{k}=C z_{1}{ }^{\prime} \cdot C z_{1}, \quad R_{k}=10^{4} \cdot I$, матрицы наблюдателя полного порядка выбраны единичными, т.е. $Q_{L}=I, R_{L}=I$.

Супервизорная система $S_{2}$. Структурная схема системы супервизорного управления $S_{2}$ представлена на рис. 4. Как и супервизорной системе $S_{1}$ для CAУ на базе LQR-регулятора объектом управления является система $S_{0}$. Однако управляющие воздействия LQR регулятора в этой системе суммируются с управляющими воздействиями ПИ регуляторов.

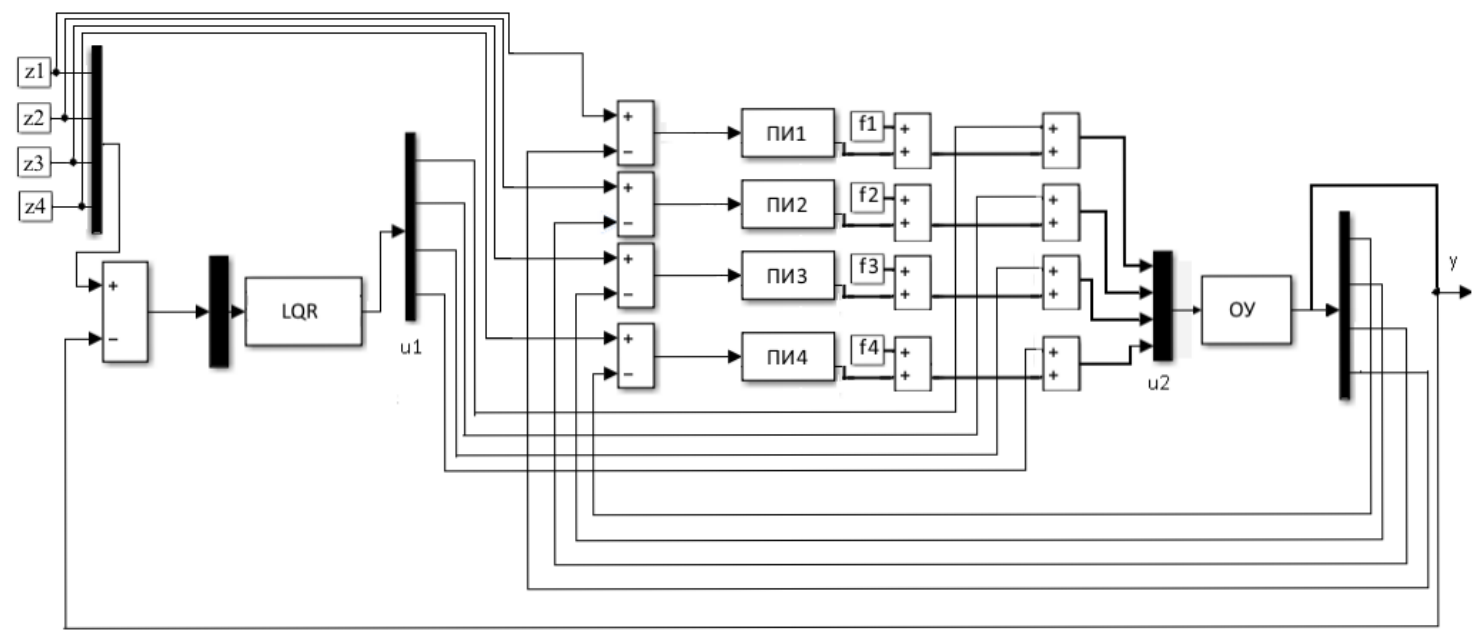

Рис. 4 - Математическая модель системы супервизорного управления $S_{2}$ 


\section{МЕТОДИ ТА АЛГОРИТМИ ЕФЕКТИВНОГО УПРАВЛІННЯ ОБ‘ЭКТАМИ}

Для LQR-регулятора объектом управления является CAУ $S_{0}$, матрицы которой имеют вид

$$
A z_{2}=\left[\begin{array}{cc}
A_{P K}-B_{P K} \cdot D_{P I} \cdot C_{P K} & B_{P K} \cdot C_{P I} \\
-B_{P I} \cdot C_{P K} & A_{P I}
\end{array}\right], B z_{2}=\left[\begin{array}{c}
B_{P K} \\
0
\end{array}\right], C z_{2}=\left[\begin{array}{ll}
C_{P K} & 0
\end{array}\right] .
$$

Матрицы ведущего LQR-регулятора представляется следующим образом

$$
A r_{2}=A z_{2}-B z_{2} \cdot K_{2}-L_{2} \cdot C z_{2}, \quad B r_{2}=L_{2}, \quad C r_{2}=K_{2}, D r_{2}=0
$$

Матрицы LQR-регулятора и наблюдателя рассчитываются с помощью стандартных Matlab - программ

$$
K_{2}=\operatorname{dlqr}\left(A z_{2}, B z_{2}, Q_{k 2}, R_{k 2}\right), \quad L_{2}=\operatorname{dql}\left(A z_{2}, C z_{2}{ }^{\prime}, Q_{L 2}, R_{L 2}\right)^{\prime} .
$$

Для достижения оптимального переходного процесса параметры LQR-регулятора выбраны в виде $Q_{k 2}=10^{-1} \cdot C z_{2}^{\prime} \cdot C z_{2}, R_{k 2}=I$, матрицы наблюдателя полного порядка выбраны единичными, т.е. $Q_{L 2}=I, R_{L 2}=I$. Статическая ошибка в системе управления устраняется интегральной составляющей ПИ регуляторов.

Супервизорная система $S_{3}$. Структурная схема супервизорной системы $S_{3}$ представлена на рис. 5 . В этой системе ведущими являются И-регуляторы, a LQR-регулятор является ведомым, т.е. рассчитывается только по модели РК. Среди регуляторов для ведущей САУ были рассмотрены И, ПИ и ПИД регуляторы, однако с точки зрения устойчивости и качества переходного процесса лучшие результаты показали именно И-регуляторы. Этот способ включения может быть рассмотрен как альтернатива включению интегратора в структуру САУ на базе LQR-регулятора в качестве модели возмущений [10].

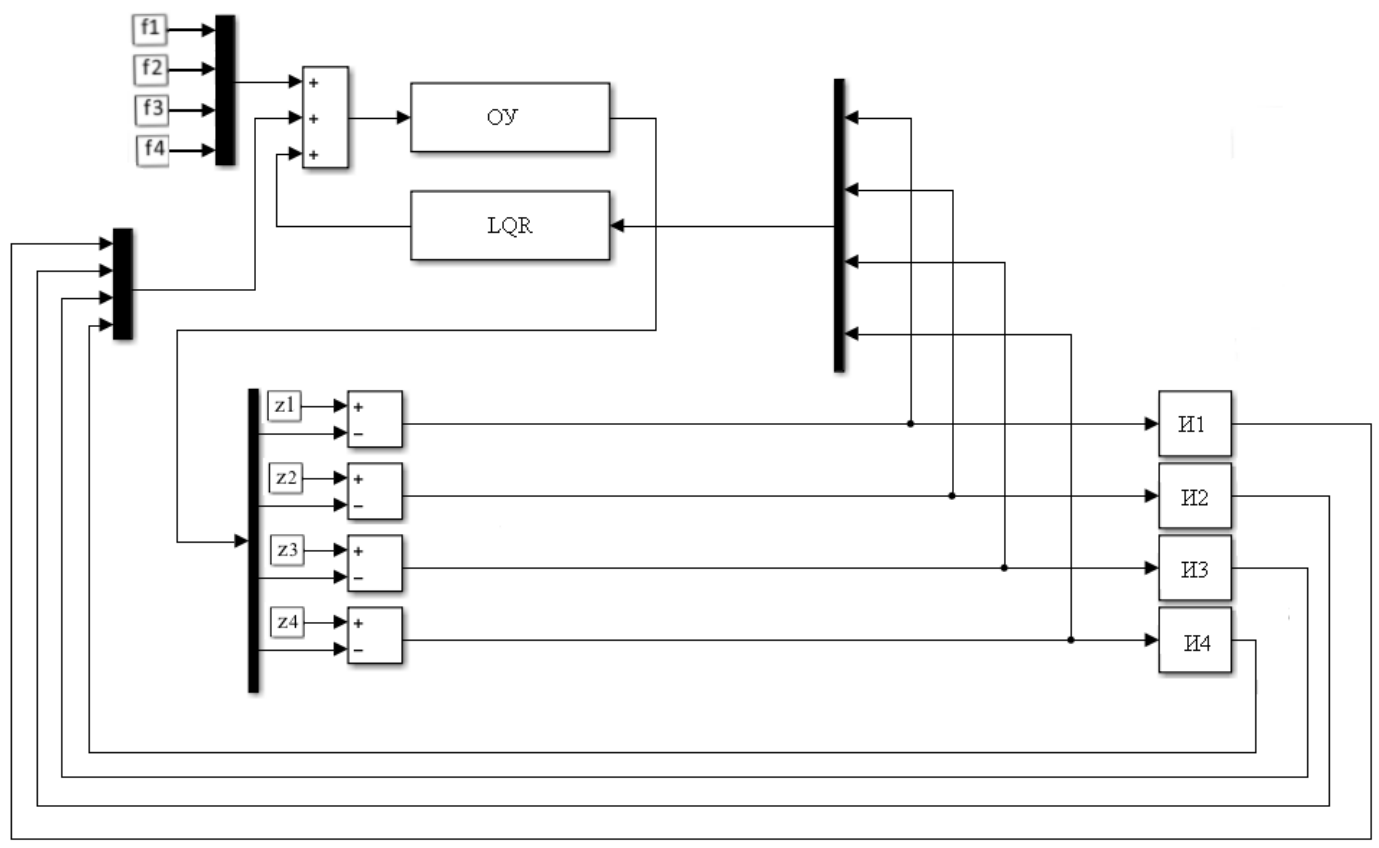

Рис. 5 - Структурная схема системы супервизорного управления $S_{3}$

Матрицы ведомого LQR-регулятора представляются следующим образом

$$
A r_{3}=A_{P K}-B_{P K} \cdot K_{3}-L_{3} \cdot C_{P K}, \quad B r_{3}=L_{3}, \quad C r_{3}=K_{3}, \quad D r_{3}=0 .
$$

где матрицы $K$ и $L$ рассчитываются с помощью реализованных в Matlab программ

$$
K_{3}=\operatorname{dlqr}\left(A_{P K}, B_{P K}, Q_{k 3}, R_{k 3}\right), \quad L_{3}=\operatorname{dqlr}\left(A_{P K}, C_{P K}{ }^{\prime}, Q_{L 3}, R_{L 3}\right)^{\prime} .
$$

Для достижения оптимального переходного процесса параметры LQR-регулятора были выбраны с использованием генетического алгоритма [9] 


\section{МЕТОДИ ТА АЛГОРИТМИ ЕФЕКТИВНОГО УПРАВЛІННЯ ОБ‘ЭКТАМИ}

$Q_{K 3}=\left[\begin{array}{cccc}646 & 0 & 0 & 0 \\ 0 & 317.339 & 0 & 0 \\ 0 & 0 & 0.0274 & 0 \\ 0 & 0 & 0 & 11.8817\end{array}\right], R_{K 3}=\left[\begin{array}{cccc}1.1117 & 0 & 0 & 0 \\ 0 & 0.5547 & 0 & 0 \\ 0 & 0 & 1.0756 & 0 \\ 0 & 0 & 0 & 31\end{array}\right]$.

Матрицы наблюдателя полного порядка выбраны единичными, т.е. $Q_{L 3}=I, R_{L 3}=I$

Настройки И-регуляторов были получены с помощью частотного метода, реализованного в Мatlab

$$
W_{I 1}=\frac{261.878 \cdot \Delta t}{2} \frac{z+1}{z-1}, \quad W_{I 2}=\frac{37.469 \cdot \Delta t}{2} \frac{z+1}{z-1}, \quad W_{I 3}=\frac{5.022 \cdot \Delta t}{2} \frac{z+1}{z-1}, \quad W_{I 4}=\frac{3.307 \cdot \Delta t}{2} \frac{z+1}{z-1} .
$$

Супервизорная система $S_{4}$. Структурная схема системы супервизорного управления $S_{4}$ представлена на рис. 6.

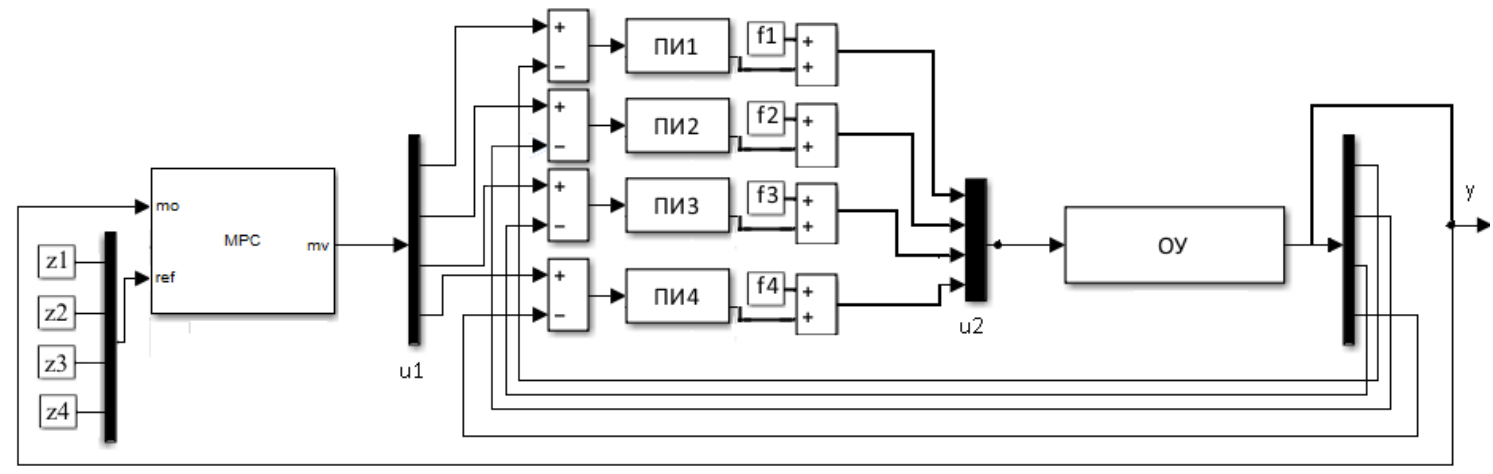

Рис. 6 - Структурная схема системы супервизорного управления $S_{4}$

Для САУ на базе МРС-регулятора объектом управления является САУ $S_{0}$, матрицы которой имеют вид

$$
A z_{4}=\left[\begin{array}{cc}
A_{P K}-B_{P K} \cdot D_{P I} \cdot C_{P K} & B_{P K} \cdot C_{P I} \\
-B_{P I} \cdot C_{P K} & A_{P I}
\end{array}\right], \quad B z_{4}=\left[\begin{array}{c}
B_{P K} \cdot D_{P I} \\
B_{P I}
\end{array}\right], \quad C z_{4}=\left[\begin{array}{ll}
C_{P K} & 0
\end{array}\right] .
$$

Реализация MPC регулятора в Мatlab является закрытой, но ряд исследований позволили её воспроизвести [8]. Заданы следующие настройки регулятора: горизонт предсказания $P=50$, горизонт управления $M=5$, коэффициент усиления фильтра оценки состояния $K F=0.2$, коэффициент усиления весовых коэффициентов $K W=0.8$, ограничения по управлению и управляемым переменным приняты в $20 \%$ от номинальных значений.

Анализ разработанных систем управления. При анализе исследовалась реакция на скачок в 5\% х.и.м, поданный одновременно на все каналы возмущения $f_{1}, f_{2}, f_{3}, f_{4}$. Переходные процессы системы представлены на рис. 7 и оценены в табл. 1. 


\section{МЕТОДИ ТА АЛГОРИТМИ ЕФЕКТИВНОГО УПРАВЛІННЯ ОБ‘ЭКТАМИ}
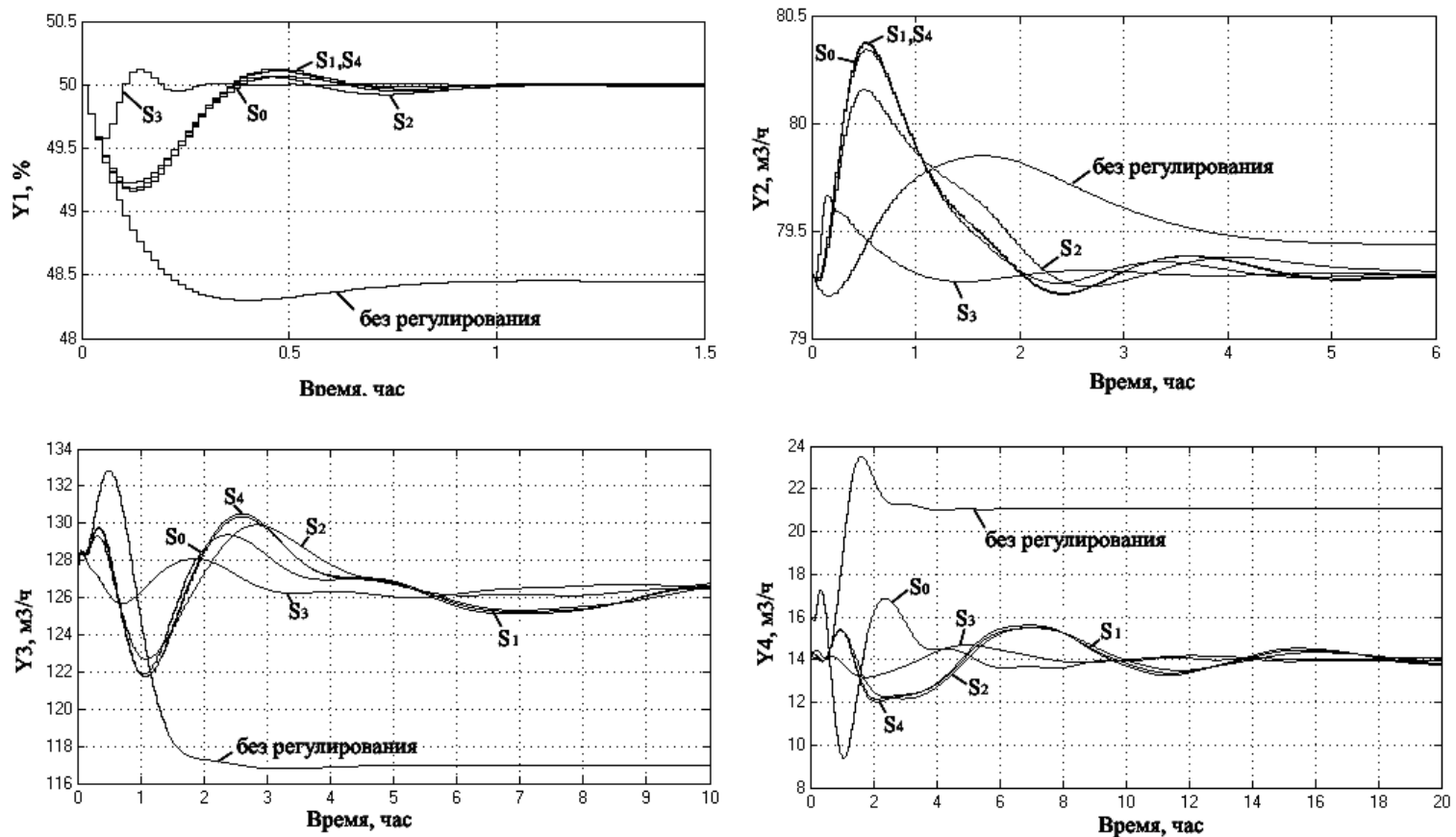

Рис. 7 - Переходные процессы при действии возмущений по нагрузке

Табл. 1 - Результаты моделирования по возмущению

\begin{tabular}{|c|c|c|c|c|c|c|c|c|}
\hline Выход & $\begin{array}{l}\text { Сис- } \\
\text { тема }\end{array}$ & $\begin{array}{l}t_{\text {уст, }}, \\
\text { час }\end{array}$ & $\mathrm{y}_{\min }\left(\mathrm{t}_{\min }\right)$ & $\mathrm{y}_{\max }\left(\mathrm{t}_{\max }\right)$ & $\begin{array}{c}\max \\
|\square \mathrm{y}| / \mathrm{Y} \\
\cdot 100 \%\end{array}$ & $\begin{array}{c}\text { Управ- } \\
\text { ление }\end{array}$ & $\begin{array}{l}\min \\
|\square \mathrm{u}|\end{array}$ & $\begin{array}{l}\max \\
|\square \mathrm{u}|\end{array}$ \\
\hline \multirow{5}{*}{$\begin{array}{c}Y_{1} \\
50 \%\end{array}$} & $\mathrm{~S}_{0}$ & 0.56 & $49.22(0.12)$ & $50.06(0.48)$ & 1.56 & \multirow{5}{*}{$\begin{array}{c}\mathrm{u}_{1} \\
50 \% \\
\text { х.и.м. }\end{array}$} & 5.71 & 0 \\
\hline & $\mathrm{S}_{1}$ & 0.87 & $49.16(0.12)$ & $50.11(0.47)$ & 1.68 & & 0.94 & 5 \\
\hline & $\mathrm{S}_{2}$ & 0.87 & $49.18(0.12)$ & $50.06(0.43)$ & 1.64 & & 0.77 & 5 \\
\hline & $\mathrm{S}_{3}$ & 0.26 & $49.58(0.05)$ & $50.12(0.13)$ & 0.84 & & 2.94 & 5 \\
\hline & $\mathrm{S}_{4}$ & 0.60 & $49.16(0.12)$ & $50.12(0.47)$ & 1.68 & & 0.97 & 5 \\
\hline \multirow{5}{*}{$\begin{array}{c}\mathrm{Y}_{2} \\
79.3 \\
\text { М3/ч }\end{array}$} & $\mathrm{S}_{0}$ & 3.51 & $79.26(2.37)$ & $80.34(0.53)$ & 1.31 & \multirow{5}{*}{$\begin{array}{c}\mathrm{u}_{2} \\
50 \% \\
\text { Х.и.м. }\end{array}$} & 5.47 & 0.09 \\
\hline & $\mathrm{S}_{1}$ & 4.06 & $79.21(2.42)$ & $80.37(0.52)$ & 1.35 & & 0.44 & 5.11 \\
\hline & $\mathrm{S}_{2}$ & 7.70 & $79.24(2.67)$ & $80.16(0.50)$ & 1.09 & & 0.38 & 5.1 \\
\hline & $\mathrm{S}_{3}$ & 1.85 & $79.26(1.45)$ & $79.67(0.15)$ & 0.47 & & 0.77 & 5 \\
\hline & $\mathrm{S}_{4}$ & 4.01 & $79.20(2.42)$ & $80.38(0.52)$ & 1.36 & & 0.49 & 5.11 \\
\hline
\end{tabular}


МЕТОДИ ТА АЛГОРИТМИ ЕФЕКТИВНОГО УПРАВЛІННЯ ОБ‘ЭКТАМИ

\begin{tabular}{|c|c|c|c|c|c|c|c|c|}
\hline \multirow{5}{*}{$\begin{array}{c}\mathrm{Y}_{3} \\
126,5 \\
\mathrm{M} 3 / \mathrm{Y}\end{array}$} & $\mathrm{S}_{0}$ & 8.36 & $121.87(1.05)$ & $129.77(0.33)$ & 3.66 & \multirow{5}{*}{$\begin{array}{c}\mathrm{u}_{3} \\
50 \% \\
\text { х.и.м. }\end{array}$} & 6.23 & 3.9 \\
\hline & $S_{1}$ & 18.16 & 121.76(1.08) & $130.36(2.62)$ & 3.75 & & 10.43 & 13.01 \\
\hline & $\mathrm{S}_{2}$ & 18.24 & $122.68(1.10)$ & 129.91(2.83) & 3.02 & & 10.51 & 11.63 \\
\hline & $\mathrm{S}_{3}$ & 9.66 & $125.68(0.72)$ & $128.52(0.07)$ & 1.60 & & 4.75 & 5 \\
\hline & $\mathrm{S}_{4}$ & 18.24 & $121.72(1.08)$ & $130.52(2.60)$ & 3.78 & & 10.85 & 13.03 \\
\hline \multirow{5}{*}{$\begin{array}{c}\mathrm{Y}_{4} \\
14 \\
\text { M3/ч }\end{array}$} & $\mathrm{S}_{0}$ & 8.36 & $9.37(1.05)$ & $17.27(0.33)$ & 33.07 & \multirow{5}{*}{$\begin{array}{c}\mathrm{u}_{4} \\
50 \% \\
\text { х.и.м. }\end{array}$} & 16.37 & 2.93 \\
\hline & $\mathrm{S}_{1}$ & 18.98 & $12.16(2.13)$ & $15.50(7.13)$ & 13.14 & & 2.27 & 27.96 \\
\hline & $\mathrm{S}_{2}$ & 18.99 & $12.17(2.95)$ & $15.48(7.03)$ & 13.07 & & 1.71 & 28.49 \\
\hline & $\mathrm{S}_{3}$ & 16.18 & $13.17(1.75)$ & $14.68(4.97)$ & 5.93 & & 1.87 & 9.1 \\
\hline & $\mathrm{S}_{4}$ & 18.92 & $12.03(2.12)$ & 15.61(6.97) & 14.07 & & 3.32 & 28.77 \\
\hline
\end{tabular}

Как видно, все рассмотренные системы управления способны устранить влияние возмущений и, следовательно, могут быть применены для управления нефтяной ректификационной колонной. Использование супервизорных систем $S_{1}, S_{2}, S_{3}, S_{4}$ позволяет получить более качественные переходные процессы по сравнению с системой $S_{0}$. Переходные процессы в системах $S_{1}$ и $S_{4}$ схожи, что объясняется близостью структуры MPC и LQRрегуляторов и общим критерием оптимальности в задаче синтеза систем на базе данных регуляторов. Самой лучшей из рассмотренных систем является супервизорная система $S_{3}$, переходные процессы в данной системе являются лучшими среди всех рассмотренных систем, как по величине максимальных отклонений, так и по времени установления.

\section{Выводы}

В мировой практике автоматизации технологических процессов вновь распространился супервизорный подход к построению систем управления сложными технологическими объектами, в основном с использованием связки САУ на базе многомерных регуляторов и САУ на базе классических регуляторов ПИД-семейства. В данной связке многомерный регулятор передает в качестве управляющих воздействий задания на контуры системы на базе децентрализованных регуляторов ПИД-семейства.

В статье исследованы возможности супервизорных систем применительно к нефтяной РК. Особенностью данного объекта управления является инерционность переходных процессов, сложная динамика каналов управления, многосвязность переменных. Качество управления колонной сильно влияет на качество конечного продукта. Как управляемые выбраны переменные, связанные непосредственно с материальным балансом.

Традиционно распространены для управления нефтяными ректификационными колоннами САУ на базе децентрализованных ПИ-регуляторов. В последнее время ряд ведущих фирм стали применять супервизорные системы с ведущей САУ на базе МРС регулятора и ведомой САУ на базе децентрализованных ПИ-регуляторов. В противовес этой стратегии управления в статье рассмотрены три более простых системы супервизорного управления:

- с передачей задания от ведущей САУ на базе LQR регулятора к ведомой САУ на базе децентрализованных ПИ-регуляторов;

- с параллельным включением CAУ на базе LQR регулятора и САУ на базе децентрализованных ПИрегуляторов;

- с параллельным включением ведущей САУ на базе децентрализованных И-регуляторов и ведомой САУ на базе LQR регулятора.

Проведенный анализ результатов математического моделирования реакции на одновременный скачок в $5 \%$ х.и.м. по всем управляющим воздействиям РК показал преимущество всех рассмотренных супервизорных систем управления по сравнению с системой на базе децентрализованных ПИ-регуляторов, параметры которых были получены с помощью частотного метода Matlab. Самой лучшей из рассмотренных систем в проведенном 
эксперименте стала супервизорная система с параллельным включением при ведущей САУ на базе И-регуляторов и ведомой CAУ на базе LQR-регулятора.

Результаты исследования рекомендуется использовать при разработке систем управления ректификационными колоннами в нефтеперерабатывающей промышленности, а также технологическими аппаратами других отраслей промышленности, для которых необходимо повышение качество управления.

Литература

[1] Tatjewski, P. Supervisory Predictive Control and on-line set-point optimization [Text] / P.Tatjewski // Int. J. Appl. Math. Comput. Sci.- 2010.- Vol. 20, No. 3 .-P. 483-495;

[2] Tatjewski,P. Advanced control of industrial processes [Text] /P.Tatjewski .- London: Springer-Verlag, 2007;

[3] Hovd, M. Modeling predictive control of a crude oil distillation column /M.Hovd, R. Micharlsen, T. Montin // Modeling, identification and control.- 1999.- Вып. 20, №. 2.- С. 75-81;

[4] Wang, Y. Fast Model Predictive Control using online optimization // Y. Wang, S. Boyd. Proc. of the $17^{\text {th }}$ World Congress. The International Federation of Automatic Control, 2008. - C.6974-6979;

[5] Skogestad, S. Control structure design for complete chemical plants / S. Skogestad // Computers and Chemical Engineering. - 2004.-Вып. 28.-С. 219-234;

[6] Технология переработки нефти. В 2-х частях. Часть первая. Первичная переработка нефти/Под. ред. О.Ф. Глаголевой и В.М. Капустина. - М.: Химия, КолосС, 2006;

[7] Веремей, Е. И. Пособие "Model Predictive Control Toolbox" [Электронный ресурс] / Е. И. Веремей, В. В. Еремеев, М. В. Сотникова. - Режим доступа: http://matlab.exponenta.ru/modelpredict;

[8] Hartley, E. Designing output-feedback predictive controllers by reverse engineering existing LTI Controller / E.Hartley, J. Maciejowski // IEEE Transactions on Automatic Control, 2013.-Вып.58.-C.2934-2939;

[9] Ghoreishi, S. Optimal Design of LQR Weighting Matrices based on Intelligent Optimization Methods / S.Ghoreishi, M. A. Nekoui, S. O. Basiri // International Journal of Intelligent Information Processing.- 2011.Vol.2.,No. 1.-C.67-74;

[10]Стопакевич, А.А. Системный анализ и теория сложных систем управления. - Одесса: Астропринт, 2013.

References

[1] Tatjewski, P. Supervisory Predictive Control and on-line set-point optimization [Text] / P.Tatjewski // Int. J. Appl. Math. Comput. Sci.- 2010.- Vol. 20, No. 3 .-P. 483-495;

[2] Tatjewski,P. Advanced control of industrial processes [Text] /P.Tatjewski .- London: Springer-Verlag, 2007.-348 $\mathrm{p}$

[3] Hovd,M. Modeling predictive control of a crude oil distillation column /M.Hovd, R. Micharlsen, T. Montin // Modeling, identification and control.- 1999.- Vol. 20, No. 2.- P. 75-81;

[4] Wang,Y. Fast Model Predictive Control using online optimization // Y. Wang, S. Boyd. Proc. of the 17th World Congress The International Federation of Automatic Control, 2008.-P.6974-6979;

[5] Skogestad, S. Control structure design for complete chemical plants / S. Skogestad // Computers and Chemical Engineering .- 2004.-Vol 28.-P. 219-234;

[6] Tehnologija pererabotki nefti. V 2-h chastjah. Chast' pervaja. Pervichnaja pererabotka nefti/Pod. red. O.F. Glagolevoj i V.M. Kapustina.- M.:Himija, KolosS, 2006;

[7] Veremej, E. I. Posobie "Model Predictive Control Toolbox" [Electronic resource] / E. I. Veremej, V. V. Eremeev, M. V. Sotnikova. - Access URL: http://matlab.exponenta.ru/modelpredict;

[8] Hartley, E. Designing output-feedback predictive controllers by reverse engineering existing LTI Controller / E.Hartley, J. Maciejowski // IEEE Transactions on Automatic Control, 2013.-Vol.58.-P.2934-2939;

[9] Ghoreishi, S. Optimal Design of LQR Weighting Matrices based on Intelligent Optimization Methods / S.Ghoreishi, M. A. Nekoui, S. O. Basiri// International Journal of Intelligent Information Processing.- 2011.Vol.2.,No. 1.-P.67-74;

[10]Stopakevich, A.A. Sistemnyi analiz i teoria slozhnyh sistem upravlenia.- Odessa: Astroprint, 2013. 